\title{
A Proposed fMRI Study: The Role of Self-Generated Object Formation in Novel Object Category Learning
}

\author{
Evin George \\ Faculty Mentor: Karin James, IU Bloomington, Department of Psychology \& Brain Sciences
}

\begin{abstract}
Recent research indicates that the self-produced visual-motor nature of handwriting provides variable visual output that better facilitates symbol understanding (Li \& James 2016). In addition, viewing novel objects/hearing novel verbs that were learned through active manipulation resulted in greater motor activation in the brain than learning through passive viewing (James \& Swain 2011). The proposed study will investigate the understanding and neural underpinnings of novel objects when learned in a self-production condition similar to handwriting: forming objects with clay. Seven- and eight-year-old participants will learn novel object categories through three conditions: a high active condition in which participants form objects with clay, a low active condition in which participants actively hold/explore pre-made objects, and a passive condition in which participants watch the experimenter hold pre-made objects. Following this training session, an object-sorting task will be used to assess the participants' knowledge of the object categories. Finally, an fMRI session will attempt to investigate motor and whole-brain activation differences between the two active conditions.
\end{abstract}

KEYWORDS: active condition; novel objects; object categories; passive condition; self-production

\section{INTRODUCTION}

$\mathrm{K}_{\mathrm{e}}^{\mathrm{n}}$ nowingly or unknowingly, we interact with our environment every day in hundreds of meaningful ways. In day-to-day life, initial sensory interaction with objects leads to subsequent neural encoding, and thus alters mental representations of object categories. The manner of the interaction itself, though, is crucial to subsequent object recognition and neural representations. The role of active learning methods and passive learning methods in object and symbol recognition have been the subject of intense study over the past twenty years. At the turn of the century, the focus narrowed to manipulating objects in virtual space. With the advent of advancing computer technology, more and more avenues for exploring the relationship between learner and object have been opened. In an experiment done in 1999, Harman, Humphrey, and Goodale found that observers who actively rotated novel three-dimensional objects performed better in object recognition than observers who passively viewed the objects. It was concluded from this result that control was key for the learner; if a participant had more control over how they learned about an object, they would be able to more quickly recognize it later (Harman et al 1999b). A separate experiment also found that active learners performed better on a perceptual match task that involved mental rotation. One potential variable introduced to the mix when manipulating objects is the impact of the preferred view/angle of the object on subsequent recognition. It was found that the "plan" views (side and front views of the object) were preferred over the intermediate views, and learning limited only to the plan views led to better performance in object recognition (James et al 2001). The use of virtual technologies to study object learning has its benefits; in particular, the researcher has a high level of control over the programming. Additionally, learning in schools is transitioning to heavy reliance on tablets and computers, making a research methodology involving technology more appropriate now than ever before. However, the major inherent drawback of these virtual technologies is the difficulty of applying the results of a virtual study to the real world.

Within the past ten years, researchers have turned to physical objects in a laboratory setting when comparing active and passive learning methods. To investigate the neural activation patterns between interacting with objects actively and passively, a 2011
fMRI study exposed young children to novel verbs and objects by having them either actively explore the object or passively watch the experimenter interact with the objects. Each novel verb, which was a given a name such as "sprocking," was paired with a 3D object which had a natural associated action, such as pulling out a retractable cord. Within the active and passive groups, participants either heard a verb or saw a photograph of the associated object while in the scanner. Participants who actively explored the objects were found to have greater motor activation in the left precentral gyrus both when hearing the novel verbs and when seeing the objects (James \& Swain 2011). Other studies have focused on the various factors that influence object recognition. For example, 1824 month old children with more expansive vocabularies performed better on object recognition than those with smaller vocabularies. Additionally, object view is once again an important factor: children preferentially opted for planar views of objects when holding them (James et al 2014).

One area of research that could shed light on some of the facets of passive and active object learning is handwriting. A comparison between symbols and objects is apt; symbols can be interpreted as two-dimensional versions of objects. Additionally, when studying symbol learning, there are clear active (writing the symbols) and passive (viewing the symbols) counterparts to object learning. A 2016 study investigated different learning techniques (visual-motor and visual-auditory) for learning novel Greek symbols. Regardless of visual-motor production, all conditions that involved learning highly variable instances of the symbols led to better symbol categorization. Because the act of handwriting produces variable forms of a letter or symbol, it helps the writer to develop a wider letter/symbol category, thus learning the letter/symbol better ( $\mathrm{Li}$ \& James 2016). The visual-motor production of handwriting has also been shown to change visual processing in the brain. Increased BOLD activation in the visual association cortex occurred during letter perception of previously handwritten letters (James 2010).

In cognitive psychology and neuroscience experiments thus far, the most active form of learning objects has been to hold and explore an object in one's hand. However, in much the same way that during handwriting one self-produces a symbol/letter, might there be a way to experimentally examine the self-production of objects? In other 
words, is it possible to "learn-by-doing" with objects (James 2010)? There could be direct analogs between symbol studies and object studies. Symbol studies have handwriting; the direct comparison to object studies may be producing objects with molding clay. With molding clay, a participant can actively create an object themselves by either using an existing object as a template or by forming a new object that might fit in an already-existing object category. How will an active object-forming condition compare to the previouslyaccepted active condition of holding an object in one's hand? This proposed experiment tests the hypothesis that forming objects with molding clay might lead to a wider mental representation of the object category than holding objects or passively viewing objects. Furthermore, the proposed experiment seeks to examine the differences in motor or whole-brain activation between the two active conditions.

\section{PROPOSED METHODS}

We hope to test 36 participants between the ages of seven years and eight years. All participants will be right-handed with normal or corrected-to-normal vision, and with no neurological compromise.

\section{General Procedure}

Participants will undergo a training session, followed by a test session, and then a fMRI session. The training and test sessions will be performed outside of the fMRI environment. The training session will have three conditions: two active, one passive. Because the study is a within-subjects design, all participants will be exposed to all three conditions. Only the order of the training conditions will differ among subjects.

The first active condition involves low visual-motor interaction in which participants are first exposed to the objects by viewing the objects and saying the category names of the objects as an experimenter holds the objects and rotates them, spending an equivalent amount of time in each planar view. Then participants will actively interact with the objects by holding and feeling the objects, given an equal amount of time for each object. The second active condition involves high visual-motor interaction in which participants are first exposed to the objects in the same way as the first condition (through observing the experimenter hold and rotate the objects) but then they are allowed to actively create and form an object with molding clay that they think would fit into the object categories. The third condition is a control condition in which participants only have an audio-visual interaction with the objects; they simply view and say the category names of the objects as the experimenter holds the object and rotates it.

After the training session, participants will be tested on their knowledge of the object categories using an object-sorting task. This task will aim to assess how participants in different conditions view the object categories. During the sorting task, participants will be asked to sort cards with pictures of objects different from the training objects into the three learned categories. There will also be cards with objects from a fourth, unlearned category, with a corresponding option for "does not belong."

After the test session, an fMRI session will be performed to test (1) whether the motor cortex will be active when the object category name is heard (auditory perception) and/or when the object is seen (visual perception); and (2) how the motor system activations differ between the two active conditions. As a control condition, participants will be exposed to pictures of novel objects without categories not seen during training, and audio clips of random words.

\section{Stimuli}

The training stimuli consist of novel three-dimensional dried clay objects, painted in monochromatic primary colors (see Figure 1). There are three learned object categories, and five physical objects in each category, making for a total of 15 clay objects for training. In each learning condition, participants will encounter all five objects from a category. Objects in the different learning conditions will be counter-balanced among participants. All the participants, regardless of condition, will learn the three categories in the same order. The order of conditions assigned to each category will be randomized.

The testing stimuli consist of cards with pictures of novel threedimensional objects, which are instances of the learned categories. There will also be pictures of objects from an additional, unlearned category. Each category will have five objects, making for a total of 20 cards.

The fMRI stimuli consist of pictures of the training objects; there will be 15 pictures for the 15 clay objects. Participants will also be shown 5 pictures of unlearned, uncategorized objects, as a control. There will also be audio recordings of five people saying the object category names, for a total of 15 recordings. Finally, there will be an additional five recordings of random words read by the five people, also as a control.

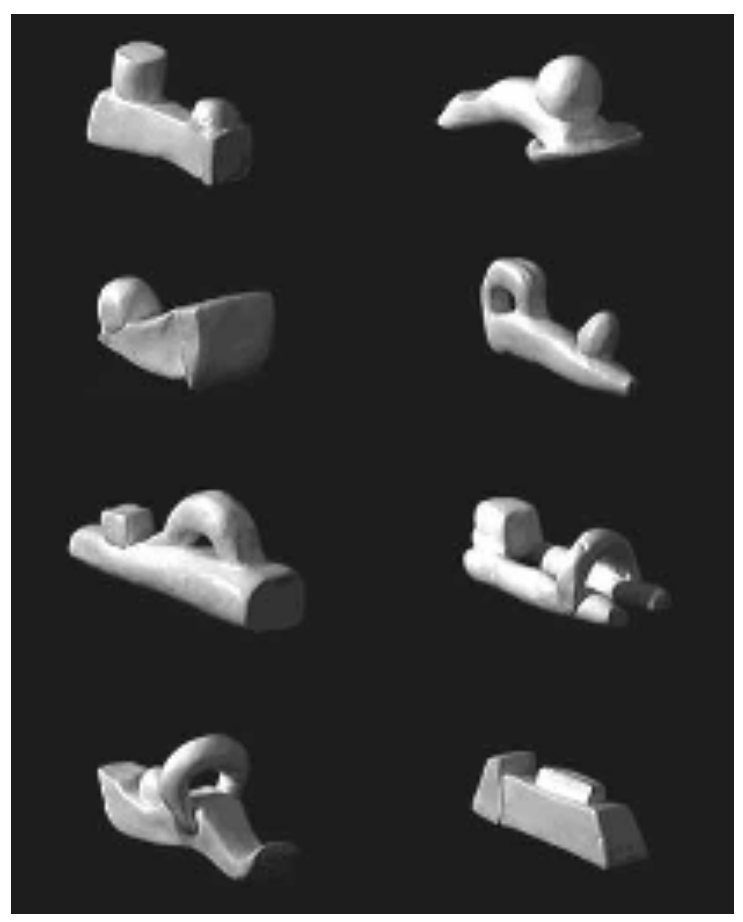

Figure 1.

Examples of the novel objects used in the propsed study (Harman \& Humphrey 1999a)

\section{EXPECTED RESULTS}

The proposed study has not been run and thus no data is available. However, based on current literature, there is a set of predictions that can logically be inferred. Because reproducing a symbol through handwriting yields several variable instances of that symbol, and learning through studying variable forms of symbols leads to more accurate symbol categorization (Li \& James 2016), it is expected that in the proposed experiment the clayforming condition will have the widest and most accurate view 
of the object categories. The clay-forming condition will generate the most variable visual output, and thus will lead to better performance on the sorting task than the other two conditions. In terms of fMRI activations, the present study is entering uncharted territory. One might expect forming objects with clay to have similar neural activations as the self-produced action of handwriting, but there are several inherent differences between the two actions that might indicate otherwise. In regard to active object interaction when compared to passive object interaction, there has been shown to be greater activation in the right superior frontal gyrus, the left middle frontal gyrus, the inferior parietal lobule (bilaterally), and the left precentral gyrus (James \& Swain 2011). Because these activations apply to a condition in which participants actively explored objects in their hands, the clayforming condition, a more involved active interaction, may have augmented activations in these areas and/or activations elsewhere in the frontal cortex.

\section{DISCUSSION AND CONCLUSION}

If the same principles governing handwriting as a self-produced action also apply to forming objects as a self-produced action, and both lead to better understanding of categories, then there are strong implications for education. If self-produced actions lead to better category understanding, regardless of what category it is, there must be a greater emphasis placed on active learning in the classroom, especially for younger children. When learning new symbol/object categories, new hands-on learning techniques like molding objects with clay or using interactive applications on tablets and computers should be emphasized. An interesting future direction for this research might be investigating the differences in word learning for young children between passive learning (seeing or hearing the words) and active learning conditions like writing the words on paper or on tablet. In the learning process, some minds have more of an inclination towards hands-on activities and some lean more towards analytical activities; however, handwriting and object research indicate that active learning leads to better understanding of symbols/objects (Li \& James 2016). Selfgenerated actions while learning may maximize motor activations in the brain and solidify category knowledge.

\section{REFERENCES}

Butler, A. J., James, T. W., \& James, K. H. (2011). Enhanced multisensory integration and motor reactivation after active motor learning of audiovisual associations. Journal of cognitive neuroscience, 23(11), 3515-3528.

Harman, K. L., \& Humphrey, G. K. (1999). Encoding 'regular'and 'random'sequences of views of novel three-dimensional objects. Perception, 28(5), 601-615.

Harman, K. L., Humphrey, G. K., \& Goodale, M. A. (1999). Active manual control of object views facilitates visual recognition. Current Biology, 9(22), 1315-1318.

James, K. H. (2010). Sensori-motor experience leads to changes in visual processing in the developing brain. Developmental science, 13(2), 279-288.

James, K. H., \& Swain, S. N. (2011). Only self-generated actions create sensori-motor systems in the developing brain.

Developmental science, 14(4), 673-678.
James, K. H., Humphrey, G. K., \& Goodale, M. A. (2001). Manipulating and recognizing virtual objects: Where the action is. Canadian Journal of Experimental Psychology/Revue canadienne de psychologie expérimentale, 55(2), 111.

James, K. H., Humphrey, G. K., Vilis, T., Corrie, B., Baddour, R., \& Goodale, M. A. (2002). "Active" and "passive" learning of three-dimensional object structure within an immersive virtual reality environment. Behavior Research Methods, Instruments, \& Computers, 34(3), 383-390.

James, K. H., Jones, S. S., Smith, L. B., \& Swain, S. N. (2014). Young children's self-generated object views and object recognition. Journal of Cognition and Development, 15(3), 393-401.

Li, J. X., \& James, K. H. (2016). Handwriting generates variable visual output to facilitate symbol learning. Journal of Experimental Psychology: General, 145(3), 298. 
